\title{
High Strain Rate Properties of High Strength Steel Sheets
}

\author{
Akihiro Uenishi $^{1)}$ Hiroshi Yoshida $^{2)}$ Shigeru Yonemura $^{3)} \quad$ Shunji Hiwatashi $^{4)}$ Satoshi Hirose $^{5)}$ Noriyuki Suzuki $^{(6)}$ \\ 1)-6) Nippon Steel Corporation \\ 20-1 Shintomi, Futtsu-City, Chiba, 293-8511, Japan (E-mail: uenishi.akihiro@nsc.co.jp)
}

Received on August 1, 2011

Presented at the JSAE Annual Congress on May 20, 2009

\begin{abstract}
High strain rate properties of sheet steel largely depend on its strength level and strengthening mechanisms. The strain rate sensitivity of flow stress has a close relation with the hardness of a softer phase, which indicates superior strain rate sensitivity of multi-phase steels such as DP and TRIP steels. Simple-shear tests have revealed an increase of the flow stress with strain even for ultra high strength steel at large strains. Based on these experimental evidences and physical understandings, an appropriate constitutive model for crash simulation is proposed and discussed.
\end{abstract}

KEY WORDS: (Standardized) materials, iron and steel materials, high-strength steel, modeling,

(Free) mechanical property,crashworthiness, high strain rate, thermally activated processes [D2]

\section{Introduction}

The demand for increased vehicle safety has become a matter of considerable concern of users, which tends to increase the car body weight. On the other hand, responding to the worldwide consciousness of environmental protection, improvement of automobile fuel consumption is also an important challenge. A light weight body with enhanced vehicle safety requires an optimum combination of body structure and material.

Numerical simulation increases its importance in designing a whole system, by clarifying the functions of each component and by choosing appropriate materials. Sheet steel products constitute the principal material used in an automobile structure, and recently, application of high strength steel has been rapidly expanded both for vehicle safety and for light weight body. Maximum strain rate during crash events is considered to be of the order of $1,000 \mathrm{~s}^{-1}$, while strain rate during press-forming is generally in the range from $0.001 \mathrm{~s}^{-1}$ to $10 \mathrm{~s}^{-1}$, and materials undergo severe plastic strain in some areas. Thus, it is crucial to consider the material behavior at large strains in a wide range of strain rate for numerical simulation.

In the present study, firstly, the relation between strain rate sensitivity of the flow stress and material strengthening is investigated by using various types of steel sheets. Generally, material strengthening is achieved by introducing heterogeneities into a material matrix. Various types of strengthening methods such as solid solution hardening, precipitation hardening, grain refinement, and second-phase hardening are utilized in commercial high strength steels. In this study, special emphasis is placed on the analysis of the relationship between the strain rate sensitivity of the flow stress and strengthening of the ferrite phase, which is the main phase of sheet steel for automobile applications. Secondly, material behavior at large strains is measured by simple shear tests, which is usually difficult to evaluate by tensile tests due to the onset of necking phenomenon. In addition, a thermomechanical analysis of the high strain rate tensile test is conducted to convert the experimental force-elongation data into true stress- true strain curves. Finally, based on the experimental and numerical results, a constitutive model suitable for crash simulation is proposed and discussed.

\section{Experimental procedure}

\subsection{Materials}

The base material (Interstitial Free steel) was Fe alloyed with about 0.2 mass $\%$ Ti to remove interstitial atoms from the ferrite matrix. Binary alloys were made by nominal addition of $1 \%$ or $2 \%$ by weight of $\mathrm{Si}$ and $\mathrm{Mn}$ to this base. Table 1 shows the composition of materials. The materials were hot-rolled to $2 \mathrm{~mm}$ and annealed at $700^{\circ} \mathrm{C}$ for 60 minutes. The average grain size was about $50 \mu \mathrm{m}$ for all materials, although it slightly decreased with the addition of solute atoms. It has already been shown that an increase of the flow stress in these materials is mainly due to the hardening by $\mathrm{Mn}$ or $\mathrm{Si}^{(1)}$. In addition to these solid solution hardened steels, commercial sheet steel materials with various strengthening mechanisms from mild steel to steel with $1180 \mathrm{MPa}$ ultimate tensile strength were used.

Table 1 Chemical composition of solid solution hardened steels (mass\%)

\begin{tabular}{lccccc}
\hline & $\mathrm{C}$ & $\mathrm{Si}$ & $\mathrm{Mn}$ & $\mathrm{Al}$ & $\mathrm{Ti}$ \\
\hline IF & 0.0016 & - & 0.10 & 0.003 & 0.015 \\
+1at.\%Mn & 0.0013 & 0.014 & 0.97 & 0.003 & 0.014 \\
+2at.\%Si & 0.0014 & 0.98 & 0.10 & 0.003 & 0.019 \\
+4at.\%Si & 0.0012 & 1.97 & 0.10 & 0.004 & 0.023 \\
\hline
\end{tabular}

\subsection{Mechanical tests}

The stress-strain curves at strain rates higher than $100 \mathrm{~s}^{-1}$ were measured by using the one bar method high strain rate tensile test (2). The method features the use of a long output bar for avoiding the influence of reflection and interference of stress waves, which are often seen with short load cell. The measurement can be finished before the arrival of the stress waves reflected at the 
other end, for the bar has a sufficient length in this method. An ordinary screw-driven test machine was used for lower strain rates.

A planar simple shear test machine ${ }^{(3),(4)}$ was used in order to characterize material behavior at large strains. In this method, simple shear deformation can be applied to the central part of the specimen by giving a displacement to one end while constraining the other end of the specimen, as shown in Fig. 1. A planar simple shear test does not involve strain inhomogeneities nor specimen necking that is inevitable in a tensile test. Thus, it is possible to measure work hardening behavior at large strains.

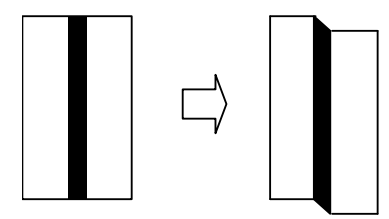

Fig. 1 Specimen $(30 \mathrm{~mm} \times 18 \mathrm{~mm})$ for a planar simple shear test before and after deformation.

\section{High strain rate properties of sheet steels}

The variation of the flow stress with strain rate in solid solution hardened steels, evaluated at 5\% strain, is shown in Fig. 2. By the addition of solute atoms, the flow stress at the lowest strain rate increases, whereas at higher strain rates its effects become less pronounced. In particular, the flow stress of $1 \% \mathrm{Mn}$ added steel becomes lower than that of the IF steel at higher strain rates, which means that the solid solution hardening by $\mathrm{Mn}$ seems to be no longer effective at higher strain rates. In Fig. 3, the strain rate sensitivity shown as the difference in the flow stress at $5 \%$ strain between $0.001 \mathrm{~s}^{-1}$ and $1,000 \mathrm{~s}^{-1}$ is plotted against the flow stress at $5 \%$ strain at a strain rate of $0.001 \mathrm{~s}^{-1}$. Clearly, the strain rate sensitivity decreases sharply by increasing static strength. The correlation between the static strength and the strain rate sensitivity in the solution hardened steels indicates that the effects of solute atoms on the matrix in iron alloys would cause hardening at low strain rates and, at the same time, softening at high strain rates.

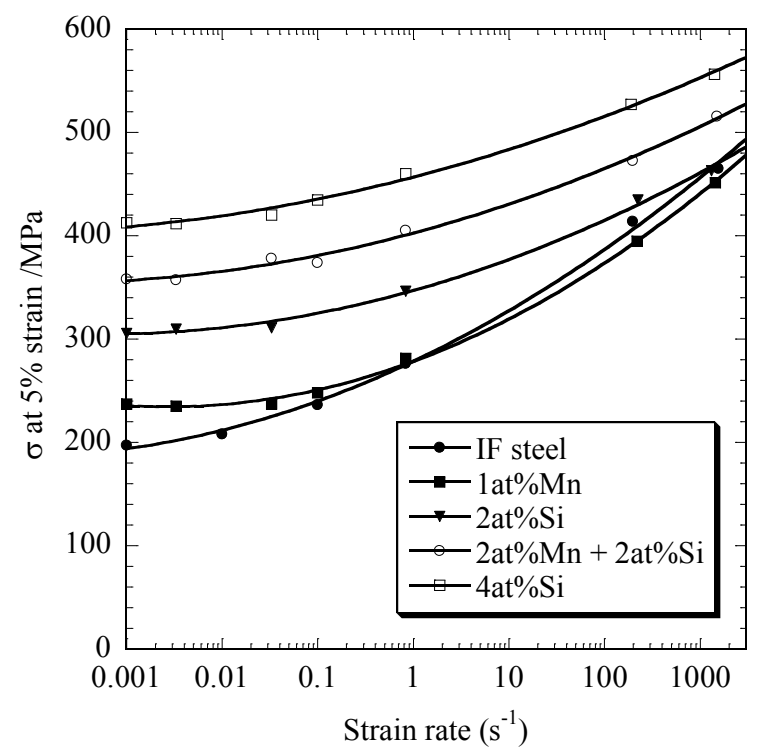

Fig. 2: Variation with the strain rate of the true tensile flow stress at $5 \%$ strain at room temperature in solution hardened steels.
A detailed investigation including thermal activation analysis was conducted to determine the mechanisms for the effects of solute atoms ${ }^{(1)}$. The result revealed that the strain rate sensitivity of the flow stress is essentially caused by a greater resistance at higher strain rate for dislocations to overcome the PeierlsNabarro potential, which has an origin in crystal structure, and the strain rate sensitivity of the flow stress in solution hardened steels is deteriorated because the misfit strain in the ferrite matrix produced by solute atoms locally changes the Peierls-Nabarro potential and promotes dislocation motion even at high strain rates. On the other hand, the misfit strain provides resistance to the overcoming of the solute atoms by dislocations, which indicates that the increase of the flow stress at the quasi-static strain rate in solution hardened steels corresponds to the magnitude of misfit strain created by solute atoms. The correlation between solid solution hardening at low strain rates and the decrease in the strain rate sensitivity at high strain rates shown in Fig. 3 is explained by considering these combined effects of solute atoms on the overall dislocation motion.

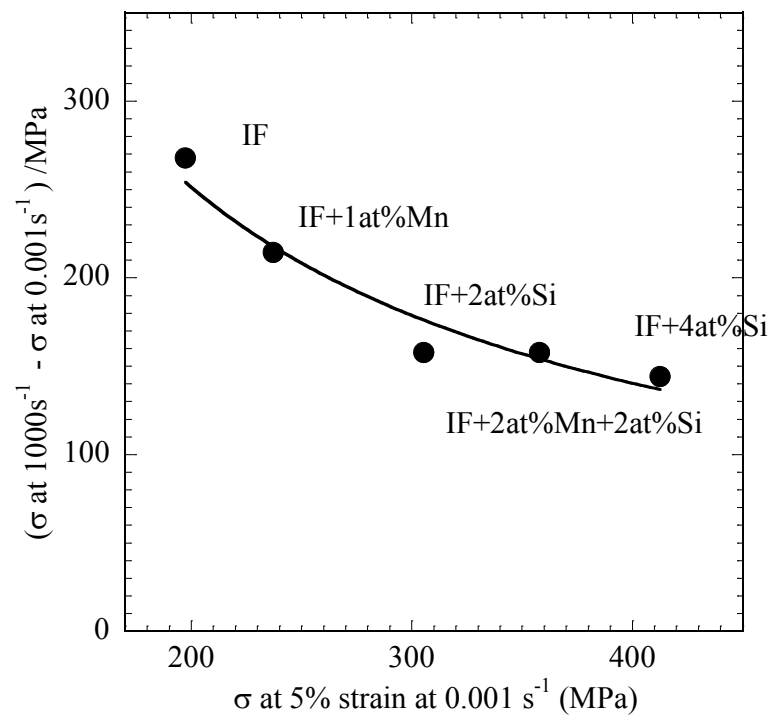

Fig. 3. Strain rate sensitivity shown as the difference in the flow stress at $5 \%$ strain between $0.001 \mathrm{~s}^{-1}$ and $1000 \mathrm{~s}^{-1}$ vs. the flow stress at $5 \%$ strain at a strain rate of $0.001 \mathrm{~s}^{-1}$.

Fig. 4 (a) shows the strain rate sensitivity of the flow stress for various sheet steels including commercial high strength steels, which was derived by the same experimental procedure for the solution hardened steels. The materials are classified into three groups: (i) solution hardened steels shown in Table 1 ( ), (ii) single-phase steels ( $\boldsymbol{\square})$ and (iii) multi-phase steels ( $\mathbf{\Delta})$. The distinction between single- and multi- phase steels was made based on the results of hardness measurement, which will be shown later. As Fig. 4 shows, there is a good correlation between the strain rate sensitivity of the flow stress and the flow stress at $0.001 \mathrm{~s}^{-1}$ for the steels with quasi-static flow stress below 600 $\mathrm{MPa}$. On the other hand, it was revealed that the flow stress at $0.001 \mathrm{~s}^{-1}$ is a poor measure of the strain rate sensitivity of the flow stress for the steels that have quasi-static flow stress greater than $600 \mathrm{MPa}$ 


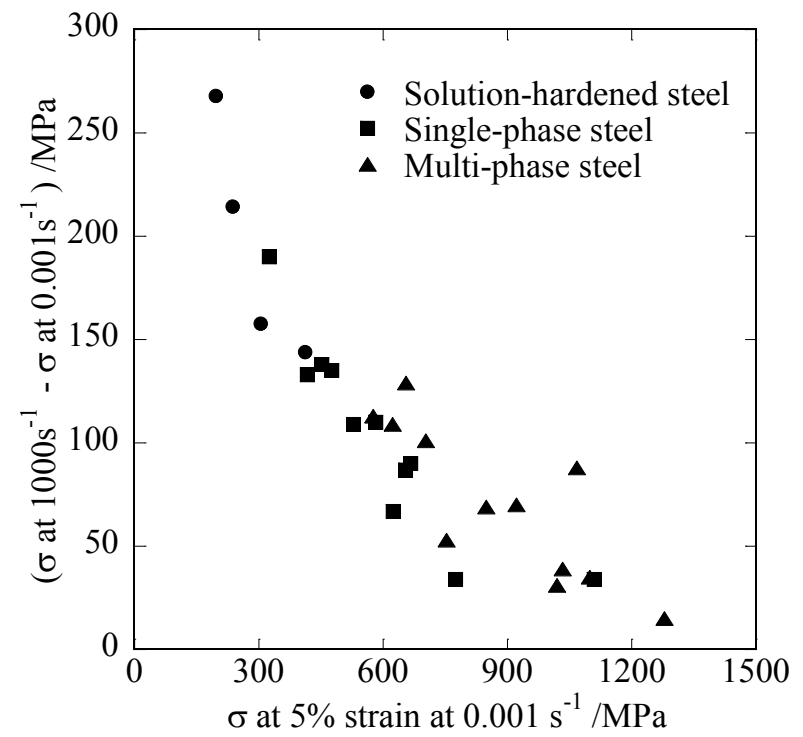

(a)

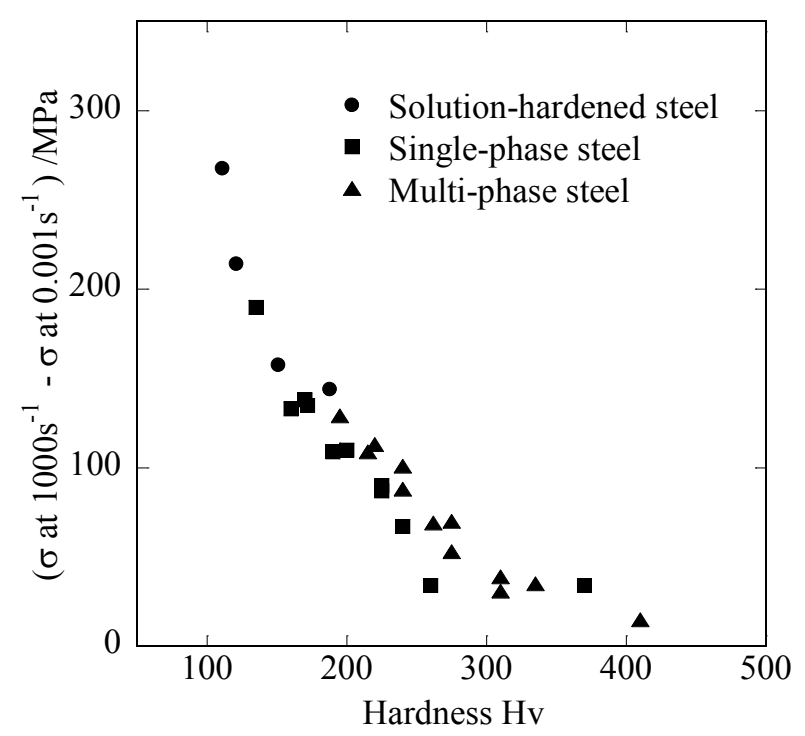

(b)

Fig. 4 Strain rate sensitivity shown as the difference in the flow stress at $5 \%$ strain between 0.001 and $1,000 \mathrm{~s}^{-1}$ vs. (a) the flow stress at $5 \%$ strain at a strain rate of $0.001 \mathrm{~s}^{-1}$ and (b) average hardness of softer phase.

In order to acquire microscopic data of materials, hardness measurement was attempted ${ }^{(5)}$. Hardness distribution of each material was obtained by executing multiple hardness measurement at intervals of $1 \mathrm{~mm}$ with small applied force of $9.8 \times 10^{-2} \mathrm{~N}$. Based on measured hardness distribution, materials presenting a single peak and those presenting multiple peaks are classified as single-phase steel and multi-phase steel, respectively (5). The results almost correspond to the classification based on microstructural observation of materials. In addition, as for the multi-phase steels, the peak hardness of the lower hardness side was evaluated. This peak hardness represents the average hardness of a softer phase and can be assumed to be that of a ferrite phase within the materials used in this study. Strain rate sensitivity of the flow stress is plotted against the evaluated hardness of the softer phase in Fig. 4 (b). As compared to Fig. 4 (a) where the flow stress at $0.001 \mathrm{~s}^{-1}$ is taken as abscissa, a strong correlation between the strain rate sensitivity of the flow stress and the hardness of a softer phase is recognized in Fig. 4 (b). This result indicates that the ferrite phase controls high strain rate properties even for the multi-phase steels. In other words, multiphase steels have superior strain rate sensitivity of the flow stress as compared to that of single-phase steels with similar quasi-static flow stress, since the ferrite phase in multi-phase steels is softer than that in single-phase steels when they have similar quasi-static strength. Therefore, materials strengthened by harder phases such as dual-phase steel and TRIP steel are suitable for impact energy absorbing applications.

\section{Work hardening behavior at large strains}

In order to assess material behavior in crash analysis, material constitutive modeling should cover not only high strain rate properties but also work hardening behavior at large strains. In general, work hardening behavior is measured by uniaxial tensile tests for flat materials. However, it is difficult to characterize the behavior at large strains in uniaxial tensile tests since an apparent flow stress decrease occurs due to the necking of the specimen after uniform elongation. Therefore, simple-shear testing was adopted. Three materials: mild steel, $590 \mathrm{MPa}$ ultimate tensile strength grade steel, and $1470 \mathrm{MPa}$ ultimate tensile strength grade steel were applied to both uniaxial tensile tests and simple-shear tests. Shear stress-shear strain curves obtained by simple shear tests were converted to equivalent tensile stress-equivalent tensile strain curves by considering the von Mises yield criterion. The results are shown in Fig. 5. At small strains, the work hardening properties measured by simple shear are almost identical to those measured by uniaxial tension, but the flow stress obtained by uniaxial tensile tests tends to decrease at larger strains. The measured decrease of the flow stress in tensile tests is due to the apparent effects of specimen necking and this apparent decrease occurs at smaller strain for materials with inferior workhardenability such as ultra high strength steels. The results obtained by simple shear tests, where the necking of the specimen does not occur, show that the flow stress continues to increase for all materials including $1470 \mathrm{MPa}$ grade steel. In crash analysis where work hardening properties at large strains are required, it is necessary to extrapolate curves before uniform elongation obtained by uniaxial tensile tests to larger strains or to apply different testing methods that can characterize behavior at large strains without apparent effects caused by geometrical instabilities of the specimen.

Large plastic strain induces heat in the deformed zone, which is proportional to the plastic work. At low strain rates, almost all heat dissipates during the deformation by thermal conduction and convection. However, at higher strain rates the deformation becomes adiabatic and the increase of temperature in the deformed zone becomes large, typically approaching a few tens of degrees ${ }^{(6)}$. Thus, the phenomenon of necking is accelerated by the thermal softening of the deformed zone at higher strain rates, although the absolute value of the softening is small by the temperature increase of a few tens of degrees. Fig. 6(a) shows stress-strain curves of mild steel obtained by tests conducted at room temperature, at constant strain rates or involving jumps in strain rate. The flow stress measured at a constant strain rate of $1,000 \mathrm{~s}^{-1}$ shows local maximum at small strain after an initial peak and then decreases at larger strains. The flow stress level after a 
strain rate jump is higher than the entirely constant strain rate curve for two different prestrains. It seems that this phenomenon is due to the early onset of necking at high strain rates ${ }^{(7)}$.

Therefore, a thermo-mechanical analysis of the high strain rate tensile test by using FEM was conducted in order to compensate the effect of necking ${ }^{(6)}$. The results of the compensations of Fig. 6(a) are shown in Fig. 6(b). The flow stress level after a strain rate jump in Fig. 6(b) is comparable to the curve at a constant strain rate, although a complete quantitative assessment is difficult, due to the difficulty of the necking compensation at large strains. It is concluded that even at high strain rates the flow stress increases with strain, as seen in the results of simple shear tests at lower strain rates.

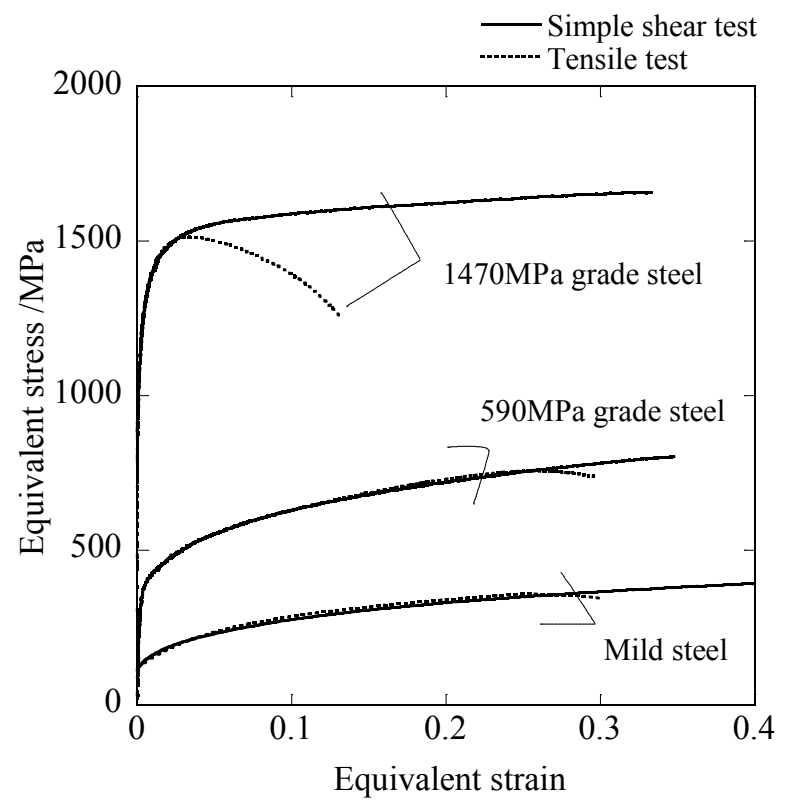

Fig. 5 Equivalent stress-strain curves of three different steels measured by simple-shear and uniaxial tensile test.

\section{Constitutive model for crash simulation}

The results obtained here can be used as a guideline for a constitutive model of high strength steel for crash application. As deformation characteristics of high strength steel, (1) the strain rate sensitivity of the flow stress depends on the strengthening mechanisms of materials and (2) the flow stress increases strain at least in the range of strain rate from $0.001 \mathrm{~s}^{-1}$ to $1,000 \mathrm{~s}^{-1}$. A number of constitutive models have been proposed for accurate material description at crash events. In any case, it should be noted that a compensation of necking effects, which are typical in uniaxial tensile tests, is necessary, regarding work hardening behavior used for parameter identification of the models. Considering limitations of present FEM codes and practical mechanical testing methods, a combination of a Swift-type equation for work hardening properties and the Cowper-Symonds empirical formula for strain rate sensitivity of the flow stress is adequate for crash simulation of high strength steels. Accordingly, the stress can be expressed by the equation below.

$$
\sigma_{e q}=K\left(\varepsilon_{0}+\varepsilon_{e q}\right)^{n}\left[1+\left(\frac{\dot{\varepsilon}_{e q}}{D}\right)^{\frac{1}{p}}\right]
$$

Here, $\sigma_{e q}, \varepsilon_{e q}$ and $\dot{\varepsilon}_{e q}$ are equivalent stress, equivalent plastic strain and equivalent plastic strain rate, respectively. $K, \varepsilon_{0}$ and $n$ are material parameters of work hardening behavior, $D$ and $p$ are those of strain rate sensitivity of flow stress.

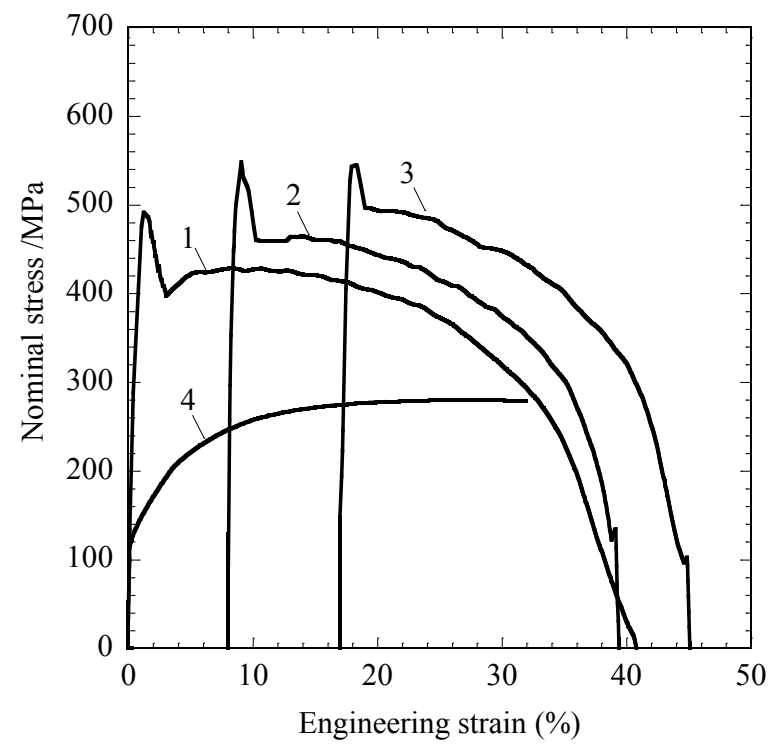

(a)

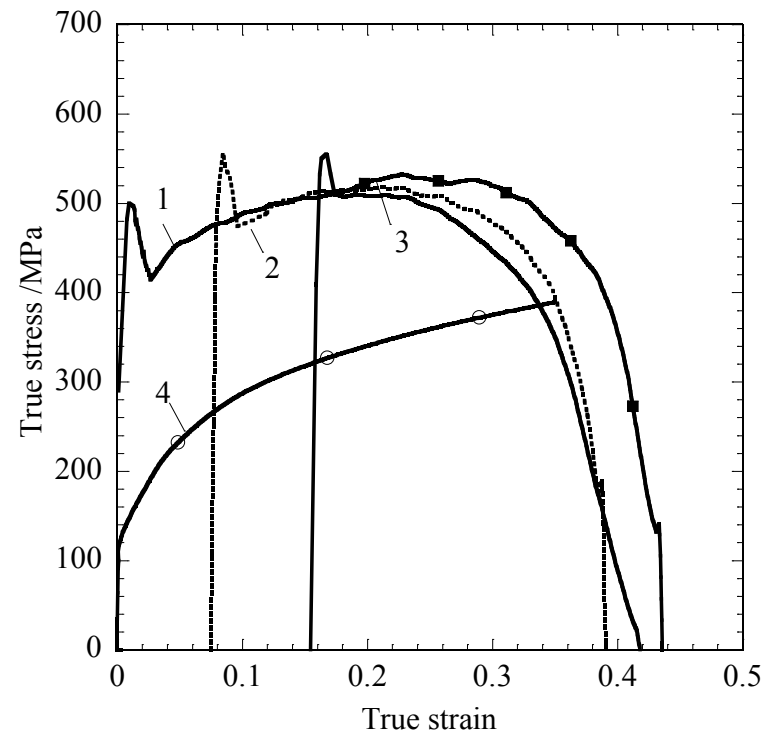

(b)

Fig. 6. Stress-strain curves of mild steel at different strain rates, including strain rate jump tests (a) before and (b) after compensations: 1) tensile test at $\left.1,000 \mathrm{~s}^{-1} ; 2\right)$ tensile test at 1,000 $\mathrm{s}^{-1}$ after a tensile prestrain of $8 \%$ at $0.001 \mathrm{~s}^{-1} ; 3$ ) after a tensile prestrain of $16 \%$ at $0.001 \mathrm{~s}^{-1} ; 4$ ) tensile test at $0.001 \mathrm{~s}^{-1}$.

\section{Conclusions}

Material behavior of high strength steel sheets at high strain rates was studied and the results are summarized as follows. 
1. Strain rate sensitivity of the flow stress decreases with quasi-static strength. This is due to the fact that obstacles for dislocations introduced to increase quasi-static strength facilitate dislocation motions at high strain rates, where the PeierlsNabarro mechanism plays an important role in increasing the flow stress.

2. Hardness of a softer phase can be used as an index of strain rate sensitivity of the flow stress in commercial high strength steel sheets. Thus, multi-phase steels such as dual-phase and TRIP steels have superior strain rate sensitivity of the flow stress as compared to that of single-phase steels with similar quasi-static flow stress, since they contain softer ferrite as a main phase.

3. In uniaxial tensile tests, it is difficult to measure work hardening properties at large strains. which becomes more critical for tensile tests at a higher strain rate because of the early necking phenomenon induced by adiabatic deformation. The results obtained by simple shear tests and compensations using FEM revealed that the flow stress increases with strain within the strain rate range examined in this study.

4. Based on the experimental and numerical investigations, appropriate material constitutive models for crash analysis were discussed.

\section{References}

(1) A. Uenishi, C. Teodosiu: Solid solution softening at high strain rates in $\mathrm{Si}$ - and/or Mn-added interstitial free steels: Acta Materialia, Vol.51, p.4437-4446 (2003)

(2) K. Kawata, S. Hashimoto, K. Kurokawa, N. Kanayama: A New Testing Method of the Characterization of Materials in High Velocity Tension: "Mechanical Properties at High Rates of Strain", ed. J. Harding, Institute of Physics, Conference Series No. 47, p.71-80 (1979)

(3) N. Suzuki, S. Hiwatashi, A. Uenishi, T. Kuwayama, Y. Kuriyama, X. Lemoine, C. Teodosiu: Advanced Constitutive Model for Spring Back Prediction of High Strength Steel Sheet: Sosei to Kakou (in Japanese), Vol.46, No.534, p.636-640 (2005)

(4) E. Nesterova, B. Bacroix, C. Teodosiu: Microstructure and Texture Evolution under Strain-Path Changes in Low-Carbon Interstitial-Free Steel: Metallurgical and Materials Transactions A Vol.32A, p.2527-2538 (2001)

(5) A. Uenishi, H. Yoshida: Effects of Soft-Phase Hardeness on Strain-Rate Sensitivity of Flow Stress in High-Strength Steeld: Sosei to Kakou (in Japanese), Vol.46, No.534, p.646-650 (2005) (6) A. Uenishi, C. Teodosiu: Constitutive modelling of the high strain rate behaviour of interstitial-free steel: International Journal of Plasticity Vol. 20 p.915-936 (2004)

(7) M. Kuroda, A. Uenishi, H. Yoshida, A. Igarashi: Ductility of interstitial-free steel under high strain rate tension: Experiments and macroscopic modeling with a physically-based consideration: International Journal of Solids and Structures Vol. 43 p.44654483 (2006) 